\title{
DEPENDENCY OF OPTIMAL PARAMETERS OF THE IRIS TEMPLATE ON IMAGE QUALITY AND BORDER DETECTION ERROR
}

\author{
I. A. Matveev ${ }^{a, b}$, V. P. Novik ${ }^{\text {b,* }}$ \\ a Dorodnicyn Computing Centre FRC CSC RAS, Vavilova, 40, Moscow, 119333, Russia - matveev@ccas.ru \\ b Iritech Inc. Moscow RnD office, B.Tatarskaya, 21, Moscow, 115184, Russia - novikvp@mail.ru
}

Commission II, WG II/10

KEY WORDS: Iris Recognition, Gabor Wavelet, Biometric Template

\begin{abstract}
:
Generation of a template containing spatial-frequency features of iris is an important stage of identification. The template is obtained by a wavelet transform in an image region specified by iris borders. One of the main characteristics of the identification system is the value of recognition error, equal error rate (EER) is used as criterion here. The optimal values (in sense of minimizing the EER) of wavelet transform parameters depend on many factors: image quality, sharpness, size of characteristic objects, etc. It is hard to isolate these factors and their influences. The work presents an attempt to study an influence of following factors to EER: iris segmentation precision, defocus level, noise level. Several public domain iris image databases were involved in experiments. The images were subjected to modelled distortions of said types. The dependencies of wavelet parameter and EER values from the distortion levels were build. It is observed that the increase of the segmentation error and image noise leads to the increase of the optimal wavelength of the wavelets, whereas the increase of defocus level leads to decreasing of this value.
\end{abstract}

\section{INTRODUCTION}

Commonly adopted work-flow of identification of human by the iris image contains several steps: obtaining eye image, segmentation of the iris region, building the iris feature set, and finally matching two such sets by a distance function. These steps were described in the very early works on iris identification (Daugman, 1993, Wildes, 1997). Therein methods of detecting stable and informative iris features were proposed also. The clue idea of iris feature extraction is convolving normalized iris image with a filter, which is local both in spatial and frequency domains. Most popular type of the filter is so called Gabor wavelet (Daugman, 1993). Its simplified 1D representations in spatial and frequency domains are

$$
\begin{aligned}
& g_{\sigma \lambda}(x)=\exp \left(-\frac{x^{2}}{2 \sigma^{2}}\right) \exp \left(-i \frac{x}{\lambda}\right) \\
& G_{\sigma \lambda}(u)=\exp \left(-\frac{\left(u-\lambda^{-1}\right)^{2} \sigma^{2}}{2}\right)
\end{aligned}
$$

where $\sigma$ and $\lambda$ determine the spread of the wavelet in spatial domain and the wavelength of modulation. Using inverted values $S=1 / \sigma$ and $W=1 / \lambda$ one can obtain simpler notation in frequency domain:

$$
G_{S W}(u)=\exp \left(-\frac{(u-W)^{2}}{2 S^{2}}\right) .
$$

The modification of this is Log-Gabor function (Field, 1987), represented in frequency domain as:

$$
G_{S W}(u)=\exp \left(\frac{-\log ^{2}(u / W)}{2 \log ^{2}(S / W)}\right)=
$$

\footnotetext{
${ }^{*}$ Corresponding author
}

$$
\exp \left(-\frac{(\log u-\log W)^{2}}{2 \log ^{2} S^{\prime}}\right)
$$

which is same as (2) except every quantity is replaced by its logarithm value. Since $S^{\prime}=S / W=\lambda / \sigma$ it represents a ratio of wavelength to spread and is used frequently instead of spread itself. Log-Gabor wavelet is also used widely: (Kumar and Passi, 2010, Masek, 2003, Peters, 2009, Al-Saqqa et al., 2013). Most likely, Log-Gabor function class is close to optimal for the given task.

Filters (1), (3) depend on two parameters, $\sigma$ and $\lambda$. Figure 1 illustrates that their variations yield extraction of different spatiofrequency features of iris image. So the choice of parameters is an important question. For the identification system, the principal task is minimizing the recognition error probability. Here it is estimated as the equal error rate (EER).

The problem of choosing $\lambda$ and $S^{\prime}$ minimizing the EER in each given system was studied by many researchers. In (Masek, 2003) optimal values of $S^{\prime}=0.5$ and $\lambda$ varies from 12 to 18 pixels. In (Peters, 2009) best performance was achieved with $\lambda=12$ $S^{\prime}=0.5$. In (Kumar and Passi, 2010) Log-Gabor filter is used, optimal parameters are determined for two databases for CASIA1 (Institute of Automation, Chinese Academy of Sciences, 2010) $\lambda=18, S^{\prime}=0.55$, for CASIA-3 $\lambda=22, S^{\prime}=0.55$. In (Al-Saqqa et al., 2013) optimal $S^{\prime}$ for different image types is found in range $[0.45,0.6]$ and wavelength is fixed $\lambda=12$. Filters of variable scale are applied for template creation in (Nabti and Bouridane, 2008). It should be noted that these works use various public and private databases, collected by various iris registration devices. Also the segmentation precision is unknown. In the work (Sagbakken, 2007) dependency of EER upon noise, defocusing and non-linear brightness modifications was studied. The influence of segmentation to error level was thoroughly investigated in (Hofbauer et al., 2016) for three databases.

However the influence of these factors to optimal shape of feature 


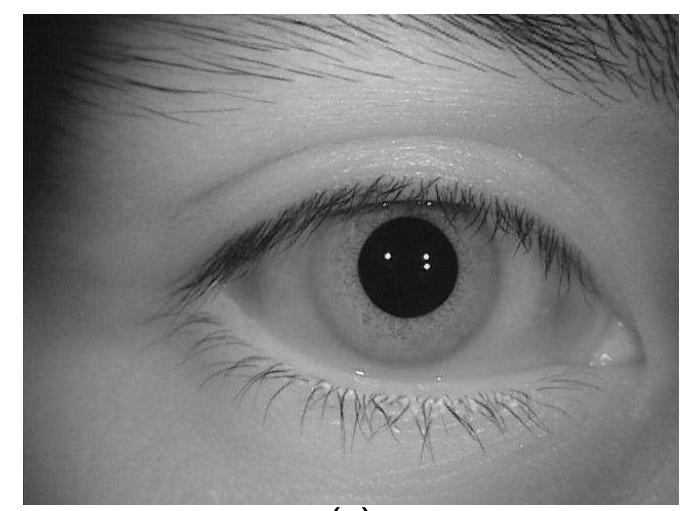

(a)

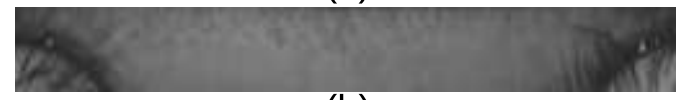

(b)

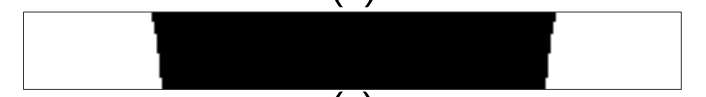

(c)

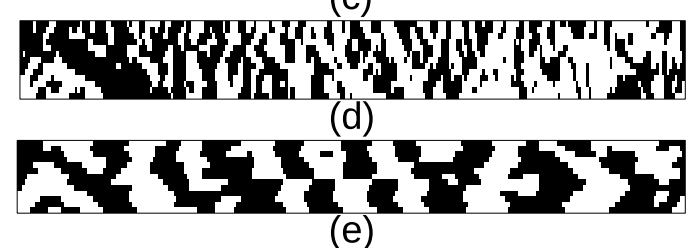

(e)

Figure 1. (a) sample of source data, (b) normalized image, (c) normalized occlusion mask, (d) binarized Log-Gabor $G_{\sigma \lambda}(u)$ with $S^{\prime}=0.2, \lambda=5$ and (e) binarized Log-Gabor $G_{\sigma \lambda}(u)$ with $S^{\prime}=0.65, \lambda=25$.

detection filter was not studied so far. In this work the attempt is made to study how segmentation precision, degree of defocusing, noise level affects EER, $\lambda$ and $S^{\prime}$ optimal values. Results for Log-Gabor filter are presented, Gabor filter behaves similarly.

\section{EXPERIMENT SETUP}

Consider a test database containing a set of eye images, like the one in Figure 1(a). Images are labelled with the persons' names in order to verify the correctness of identification. There is more than one person in the database and more than one image per person.

Each image $I(x, y)$ is processed in the following way. Iris segmentation method (Gankin et al., 2014) is applied and outputs the coordinates of pupil contour, iris contour and occlusion mask. The pupil contour is the circle represented via its center and radius $\left(x_{P}, y_{P}, r_{P}\right)$, which is the best approximation of pupil-iris boundary. The iris contour is the circle $\left(x_{I}, y_{I}, r_{I}\right)$, approximating iris-sclera boundary. The occlusion mask $M(x, y)$ is an image of same size as source with zero pixels in place where iris is covered by obstacles like eyelids, eyelashes, flashes.

Then the iris normalisation is performed. It is a transformation of a ring, enclosed between iris and pupil circles to a rectangular region. The normalized image coordinate system is rectilinear $O \phi \rho$, where horizontal axis $O \phi$ corresponds to angle measured along the pupil and iris circles in source image, and vertical axis $O \rho$ corresponds to radial shift from pupil circle to iris circle. This transformation is reduced to a well-known polar transform in case if pupil and iris centres coincide, $\left(x_{P}, y_{P}\right)=\left(x_{I}, y_{I}\right)$. Both source image $I(x, y)$ and mask $M(x, y)$ are subjected to the transformation, which yields their normalized versions $I(\phi, \rho)$ and $M(\phi, \rho)$. Figure 1(b) depicts a sample of normalized image obtained from image in Figure 1(a) and Figure 1(c) shows occlusion mask thereof.

There several possible models of this transformation, here the most popular called "rubber-sheet model" (Daugman, 2002) is used. The origin $(x, y)$ for the point of normalised image $(\phi, \rho)$ is expressed as:

$$
\begin{aligned}
& x(\rho, \phi)=(1-\rho) x_{1}(\phi)+\rho x_{2}(\phi), \\
& x_{1}(\phi)=x_{P}+r_{P} \cos (\phi), \\
& x_{2}(\phi)=x_{I}+r_{I} \cos (\phi),
\end{aligned}
$$

Coordinate $y$ is computed accordingly. Dimensions of normalized image are in ranges: $\rho \in[0 ; 1], \phi \in[0 ; 2 \pi)$. Brightness of the normalized image is obtained with the bilinear interpolation:

$$
\begin{gathered}
N(\rho, \phi)= \\
(1-\{x\})(1-\{y\}) I(\lfloor x\rfloor,\lfloor y\rfloor)+ \\
\{x\}(1-\{y\}) I(\lfloor x\rfloor+1,\lfloor y\rfloor)+ \\
(1-\{x\})\{y\} I(\lfloor x\rfloor,\lfloor y\rfloor+1)+ \\
\{x\}\{y\} I(\lfloor x\rfloor+1,\lfloor y\rfloor+1),
\end{gathered}
$$

where $\lfloor a\rfloor$ and $\{a\}$ are integer and fractional parts of $a$ respectively.

Iris features $V(\phi, \rho)$ are calculated as convolution of normalized image (5) with filters (2), (3):

$$
\begin{aligned}
V(\phi, \rho) & =N(\phi, \rho) * g_{\sigma \lambda}(\phi)= \\
& =\mathcal{F}^{-1}\left\{\mathcal{F}\{N(\phi, \rho)\} \mathcal{F}\left\{g_{\sigma \lambda}(\phi)\right\}\right\}= \\
& =\mathcal{F}^{-1}\left\{\mathcal{F}\{N(\phi, \rho)\} G_{S W}(u)\right\} .
\end{aligned}
$$

Here $\mathcal{F}$ is the Fourier transform. Finally, the features used for matching are obtained as binarization of real and imaginary parts of convolved array (6):

$$
\begin{aligned}
& T_{R e}(\phi, \rho)= \begin{cases}1, & \Re(V(\phi, \rho))>0, \\
0, & \text { otherwise, }\end{cases} \\
& T_{\text {Im }}(\phi, \rho)= \begin{cases}1, & \Im(V(\phi, \rho))>0, \\
0, & \text { otherwise, }\end{cases}
\end{aligned}
$$

Two components of (7) are joined together to form a template. So, each eye image $I$ is converted to a template $T(I)$ and accompanying mask $M(I)$.

Let set of images, or database $B=\left\{I_{1}, \cdots, I_{M}\right\}$ contain $L$ images, belonging to $P$ persons. Each person $p, p \in[1 ; P]$ is represented by several images, composing subset: $B_{p}=$ $\left\{I_{p, 1}, \cdots, I_{p, L_{p}}\right\} \subset B$, so as $\sum L_{p}=L$. Biometric template $T(I)$ is generated from each image. Any two templates can be matched with Hamming distance:

$$
d_{0}\left(T_{1}, T_{2}\right)=\frac{1}{|\Omega|}\left|\left\{T_{1}(\phi, \rho) \neq T_{2}(\phi, \rho),(\phi, \rho) \in \Omega\right\}\right|,
$$

where $\Omega=M_{1} \cap M_{2}$ is the intersection of non-occluded areas of two matching templates. In fact, more complex distance function is used, which counts on possible uncertainty of iris angle due to image rotation. The rotation of source eye image turns to cyclic shift along $\phi$ coordinate in normalized image. One of the templates (together with mask) is rotated and matched, minimum 
distance is found:

$$
\begin{aligned}
d\left(T_{1}, T_{2}\right) & =\min _{\psi} d_{\psi}\left(T_{1}, T_{2}\right), \\
d_{\psi}\left(T_{1}, T_{2}\right) & =\frac{1}{\Omega(\psi)}\left|\left\{\begin{array}{c}
T_{1}(\phi+\psi, \rho) \neq T_{2}(\phi, \rho), \\
(\phi, \rho) \in \Omega(\psi)
\end{array}\right\}\right| \\
\Omega(\psi) & =M_{1}(\phi+\psi) \cap M_{2}(\phi),
\end{aligned}
$$

The distance is normalized to the range $[0 ; 1]$. Without limitation of generality one can speak about matching images themselves: $d\left(I_{i}, I_{j}\right)=d\left(T\left(I_{i}\right), T\left(I_{j}\right)\right)$. Matching $d\left(I_{p, i}, I_{q, j}\right)$ is called genuine if $p=q$, i.e. images belong to one person, and impostor otherwise. All database images are pairwisely matched against each other forming totally $L^{2}$ distances. Total number of genuine matches is:

$$
N_{\text {genuine }}=\left|\left\{\left(I_{p, i}, I_{q, j}\right): p=q\right\}\right|=\sum_{p=1}^{P} L_{p}^{2},
$$

and total number of impostor matches is:

$$
N_{\text {impostor }}=L^{2}-N_{\text {genuine }} .
$$

Using distance as a classifier and taking threshold $\Theta \in[0 ; 1]$ one can determine the number of classification errors of the first kind (false reject) as the number of genuine matches with distance above or equal to $\Theta$ :

$$
N_{F R}(\Theta)=\left|\left\{\left(I_{p, i}, I_{q, j}\right): p=q, d\left(I_{p, i}, I_{q, j}\right) \geqslant \Theta\right\}\right| .
$$

Number of impostor matches with distance less or equal than $\Theta$ is a count of errors of the second kind (false accept):

$$
N_{F A}(\Theta)=\left|\left\{\left(I_{p, i}, I_{q, j}\right): p \neq q, d\left(I_{p, i}, I_{q, j}\right) \leqslant \Theta\right\}\right| .
$$

Relative errors are:

$$
E_{F R}(\Theta)=\frac{N_{F R}(\Theta)}{N_{\text {genuine }}}, \quad E_{F A}(\Theta)=\frac{N_{F A}(\Theta)}{N_{\text {impostor }}} .
$$

Call Detection Error Tradeoff, DET-curve a polyline passing through points $\left(E_{F R}(\Theta), E_{F A}(\Theta)\right)$. By construction

$$
E_{F R}(0)=1, E_{F R}(1)=0, E_{F A}(0)=0, E_{F A}(1)=1,
$$

hence there exists a threshold where lines do intersect:

$$
\Theta_{E E R}: E_{F R}\left(\Theta_{E E R}\right)=E_{F A}\left(\Theta_{E E R}\right)
$$

and values of relative errors of first an second kind equate. The value of error at the intersection is called equal error rate (EER).

Since EER depends on feature detection parameters, one can write $\operatorname{EER}\left(\lambda, S^{\prime}\right)$ and search the optimum:

$$
\begin{aligned}
& E E R^{*}=\min _{\lambda, S^{\prime}} E E R\left(\lambda, S^{\prime}\right), \\
& \left(\lambda^{*}, S^{*}\right)=\arg \min _{\lambda, S^{\prime}} E E R\left(\lambda, S^{\prime}\right) .
\end{aligned}
$$

Both small (i.e. near zero) and big (i.e. comparable with normalized image size) values of $\lambda$ and $S^{\prime}$ yield degenerate features and high EER values. Thus the problem of minimizing EER is feasible. The optimum in the performed experiments was located with exhaustive search. Figure 2 shows a sample result of such search. Horizontal axis corresponds to $\lambda$, vertical axis gives $S^{\prime}$, the EER value is defined by color as shown in scale on the right, and is

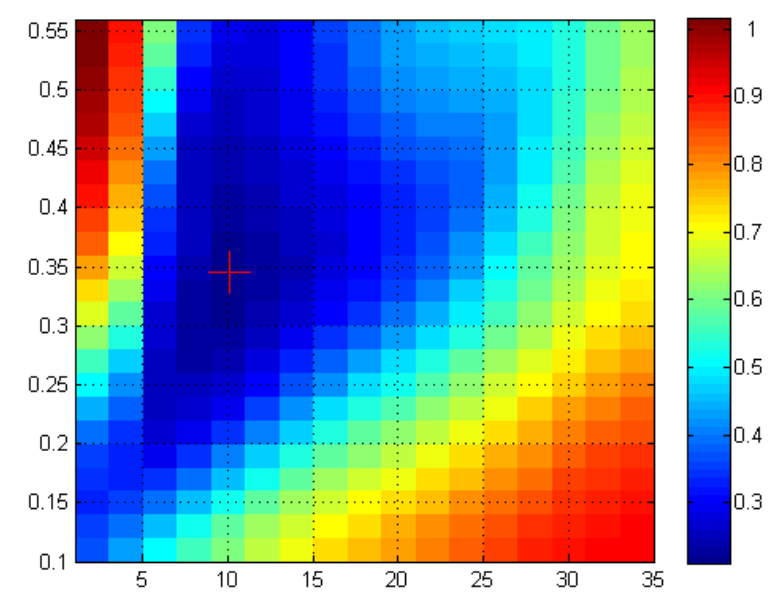

Figure 2. Search of $\lambda^{*}, S^{*}$ and $E E R^{*}$.

expressed in percent. Red cross marks the position of optimum with coordinates $\lambda^{*}=10, S^{*}=0.345$ and $E E R^{*}=0.22 \%$.

Each of examined degrading factors was modelled by introducing the controlled distortions into the source data. The strength of the distortions was set by a parameter $K_{\text {factor }}$. The dependency of $\lambda^{*}, S^{*}$ and $E E R^{*}$ on $K_{\text {factor }}$ was recorded.

Two publicly available iris image databases were used: CASIA4Thousand (Institute of Automation, Chinese Academy of Sciences, 2010), NDIRIS (Phillips et al., 2010). Hamming distance was used for matching the templates.

\section{INFLUENCE OF BLURRING AND NOISE}

Image blurring is modelled by convolution of source image with a unit filter of $3 * 3$ size, applied $K_{\text {blur }}$ times.

Figure 3 shows dependency of optimal wavelength and spread values on the blurring strength parameter $\lambda^{*}\left(K_{\text {blur }}\right)$.

One can see that optimal wavelength of Log-Gabor filter exhibit substantial decrease with the growth of blurring. This may seem strange, intuitively one would expect them growing. The explanation is the fact that Log-Gabor filter itself produce image averaging to the extent of its $\lambda$ and thus preliminary blurring plays a role of widening the wavelength, so own filter's $\lambda$ should be shifted to lower values.

Noise is modelled as an additive brightness value normally distributed with zero average and mean square deviation $K_{\text {noise }}$. Figure 4 shows dependency of optimal filter parameter values on the noise strength parameter. Increasing of the noise predictably leads to increasing EER and optimal wavelength.

\section{INFLUENCE OF SEGMENTATION ERROR}

Iris regions were segmented from image automatically by a set of methods described in (He et al., 2009, Gankin et al., 2014). Modelled distortions were applied to the images and segmentation data, then the EER values were calculated for a set of $\lambda$ and $S^{\prime}$, and the optimal values of those were detected.

Iris region is described as a ring enclosed between two (possibly non-concentric) circles, which approximate inner and outer 

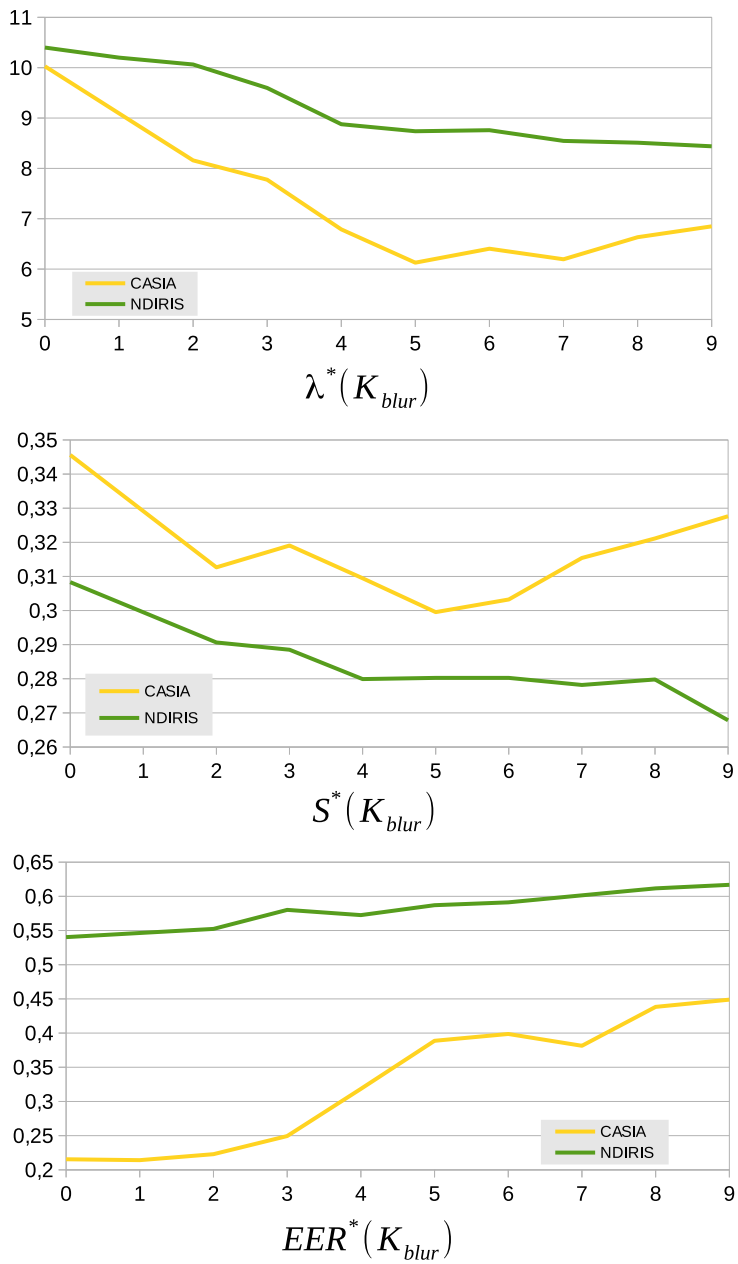

Figure 3. Dependency of optimal parameters on $K_{b l u r}$.

borders of the iris. The circles are defined by their centres and radii, $\left(x_{P}, y_{P}, r_{P}\right)$ for pupil and $\left(x_{I}, y_{I}, r_{I}\right)$ for iris. These six parameters together compose the six-dimensional vector $\overrightarrow{b_{0}}=$ $\left(x_{P}, y_{P}, r_{P}, x_{I}, y_{I}, r_{I}\right)^{T}$ for each image. Segmentation error is modelled by summing this vector with a vector $\vec{r}$ composed from independent variates uniformly distributed in a range $[-1 ; 1]$, multiplied by the parameter $K_{\text {segm }}: \vec{b}=\overrightarrow{b_{0}}+K_{\text {segm }} \vec{r}$. The parameter $K_{\text {segm }}$ determines the level of distortion.

Figure 5 shows dependency of optimal filter parameter values and EER on the segmentation distortion strength. EER is expressed in percent. The increase of $K_{\text {segm }}$ leads to growth of EER as well as optimal values of $\lambda$ and $S^{\prime}$. Thus for bigger segmentation distortions the error of recognition is higher and filters with larger wavelengths and spreads are required.

\section{CONCLUSIONS AND FURTHER WORK}

The dependency of optimal filter parameters on three data distortion types was studied.

Optimal parameters of Log-Gabor filter substantially depend upon precision of segmentation and image noise. With the increase of segmentation distortions and image noise optimal wavelength is also monotonously increasing, as well as the classification error. However, small errors of segmentation $K_{\text {segm }} \in[0 ; 2]$
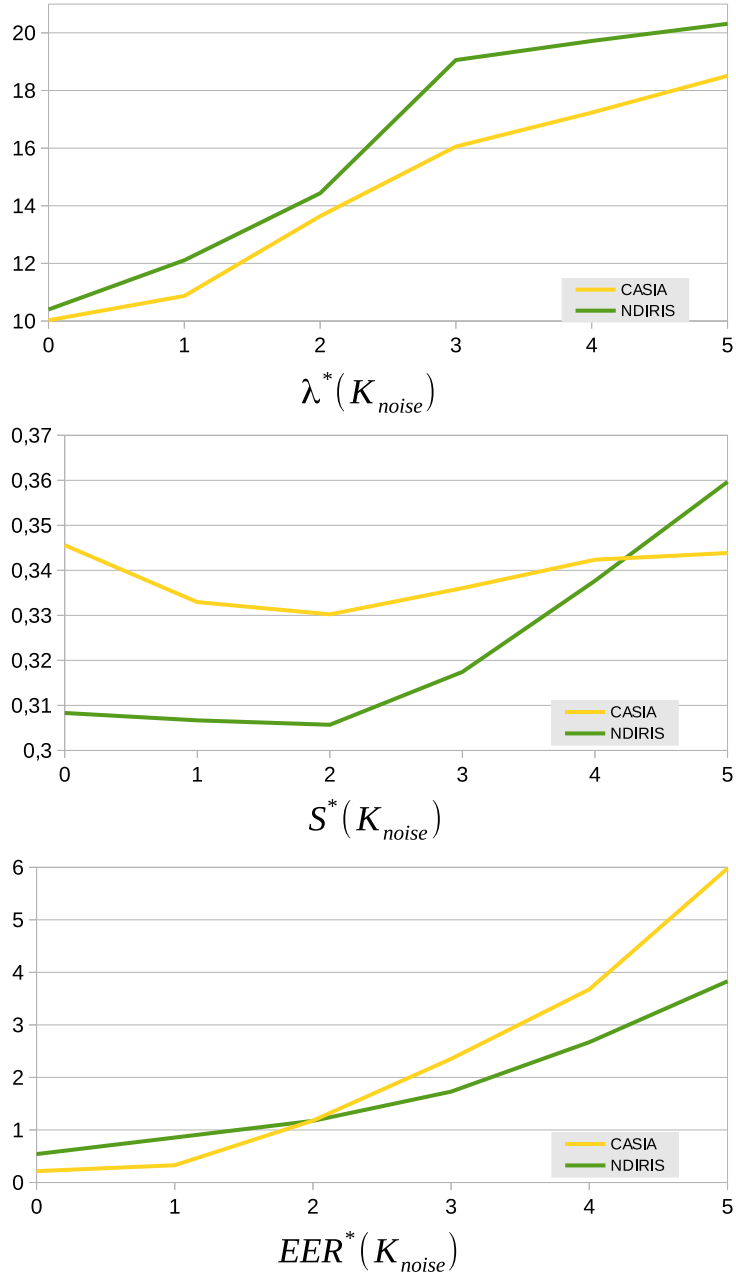

Figure 4. Dependency of optimal parameters on $K_{\text {noise }}$.

do not cause substantial change. Defocusing decreases the optimal wavelength of Log-Gabor filter.

This methodology for determining optimal parameters may be applied to any type of parametrized filters. In future work we plan to change resource-consuming exhaustive search to more elaborate optimization procedure.

\section{ACKNOWLEDGEMENTS}

The work is supported by the Russian Foundation of Basic Research, grant no. 16-07-01171.

\section{REFERENCES}

Al-Saqqa, S., Al-Rawi, M. and Al-Zoubi, M. B., 2013. Iris recognition system evaluation experiments using CASIA version 3. Journal of American Science.

Daugman, J., 2002. How iris recognition works. In: Proc. Int. Conf. Image Processing, Vol. 1, pp. I-33-I-36.

Daugman, J. G., 1993. High confidence visual recognition of persons by a test of statistical independence. IEEE Transactions on Pattern Analysis and Machine Intelligence 15(11), pp. 11481161. 

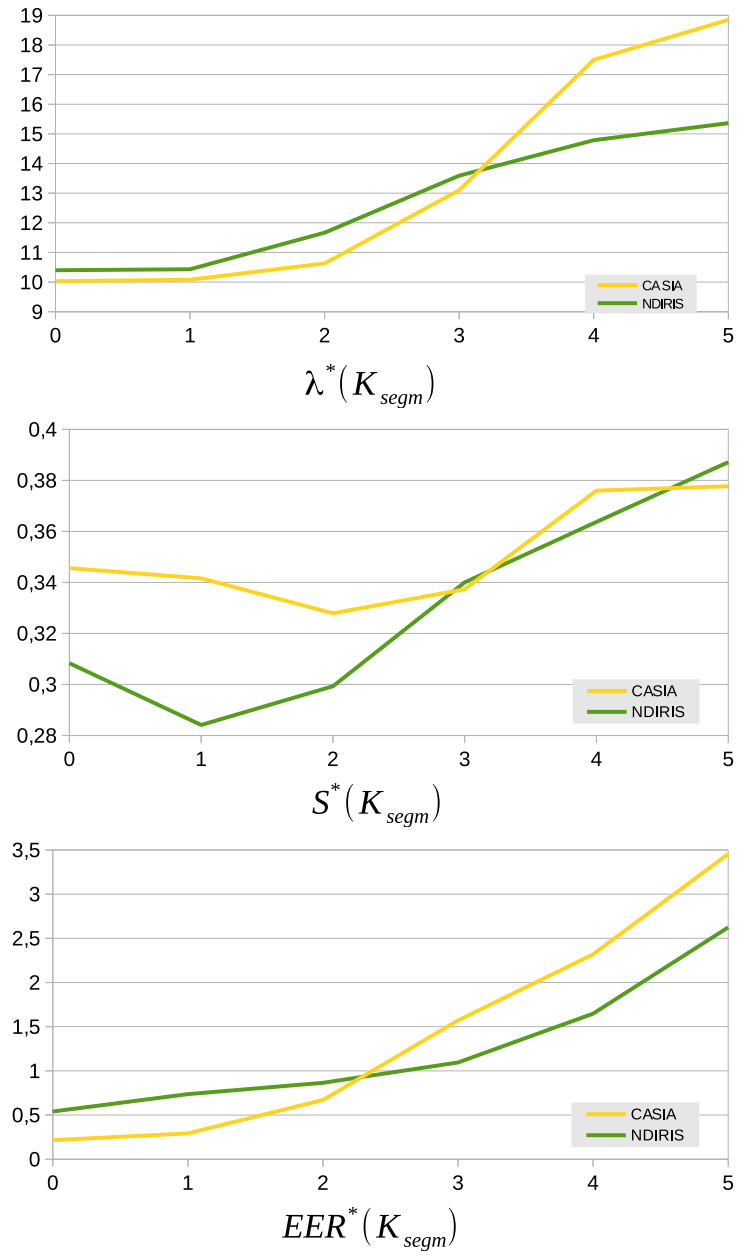

Figure 5. Dependency of optimal parameters on $K_{\text {segm }}$.

Field, D. J., 1987. Relations between the statistics of natural images and the response properties of cortical cells. J. Opt. Soc. Am. A 4(12), pp. 2379-2394.

Gankin, K., Gneushev, A. and Matveev, I., 2014. Iris image segmentation based on approximate methods with subsequent refinements. Journal of Computer and Systems Sciences International 53(2), pp. 224-238.

He, Z., Tan, T., Sun, Z. and Qiu, X., 2009. Toward accurate and fast iris segmentation for iris biometrics. IEEE Transactions on Pattern Analysis and Machine Intelligence 31(9), pp. 1670-1684.

Hofbauer, H., Alonso-Fernandez, F., Bigun, J. and Uhl, A., 2016. Experimental analysis regarding the influence of iris segmentation on the recognition rate. IET Biometrics 5(3), pp. 200-211.

Institute of Automation, Chinese Academy of Sciences, 2010. Casia iris image database.

Kumar, A. and Passi, A., 2010. Comparison and combination of iris matchers for reliable personal authentication. Pattern Recognition 43(3), pp. 1016 - 1026.

Masek, L., 2003. Recognition of human iris patterns for biometric identification. Technical report, The University of Western Australia.

Nabti, M. and Bouridane, A., 2008. An effective and fast iris recognition system based on a combined multiscale feature extraction technique. Pattern Recogn. 41(3), pp. 868-879.
Peters, T. H., 2009. Effects of segmentation routine and acquisition environment on iris recognition.

Phillips, P. J., Scruggs, W. T., O’'Toole, A. J., Flynn, P. J., Bowyer, K. W., Schott, C. L. and Sharpe, M., 2010. Frvt 2006 and ice 2006 large-scale experimental results. IEEE Transactions on Pattern Analysis and Machine Intelligence 32(5), pp. 831-846.

Sagbakken, H. C., 2007. Iris recognition under various degradation models.

Wildes, R., 1997. Iris recognition: an emerging biometric technology. Proceedings of the IEEE 85(9), pp. 1348-1363.

Revised 31st March 2017 